\title{
WATER-QUALITY AND GROUND-WATER-LEVEL DATA, BERNALILLO COUNTY, CENTRAL NEW MEXICO, 1995
}

\author{
By Dale R. Rankin
}

\section{U.S. GEOLOGICAL SURVEY}

Open-File Report 96-578

Prepared in cooperation with the BERNALILLO COUNTY ENVIRONMENTAL HEALTH DEPARTMENT

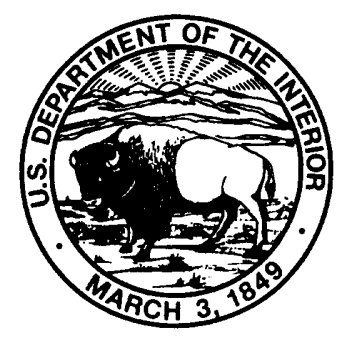

Albuquerque, New Mexico 


\title{
U.S. DEPARTMENT OF THE INTERIOR BRUCE BABBITT, Secretary
}

\author{
U.S. GEOLOGICAL SURVEY
}

Gordon P. Eaton, Director

The use of firm, trade, and brand names in this report is for identification purposes only and does not constitute endorsement by the U.S. Geological Survey

For additional information write to:

District Chief

U.S. Geological Survey

Water Resources Division

4501 Indian School Road NE, Suite 200

Albuquerque, NM 87110-3929
Copies of this report can be purchased from:

U.S. Geological Survey

Branch of Information Services

Box 25286

Denver, CO 80225-0286 


\section{CONTENTS}

Page

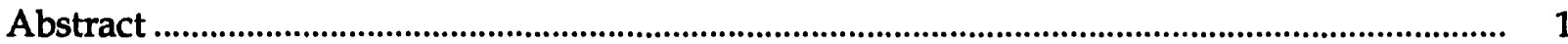

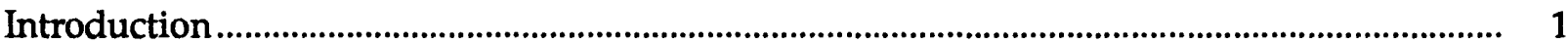

Purpose and scope

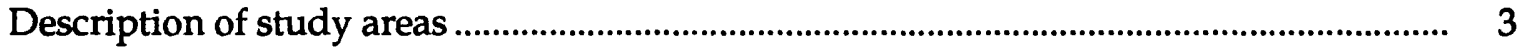

Acknowledgments ................................................................................................................ 3

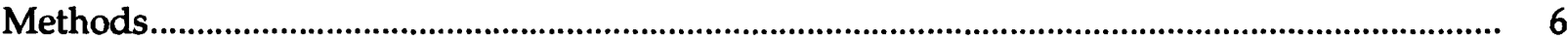

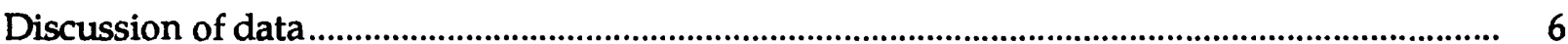

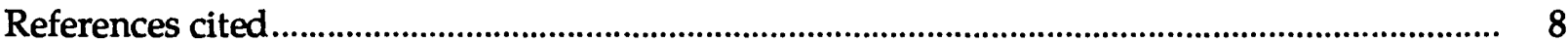

\section{FIGURES}

Figures 1-3. Maps showing:

1. Location of study areas, Bernalillo County, New Mexico ................................... 2

2. Location of wells, springs, and acequia sampled in the east mountain area, Bernalillo County, New Mexico.

3. Location of wells sampled in the northeast area, Bernalillo County, New Mexico

\section{TABLES}

Table 1. Water-quality and ground-water-level data for wells, springs, and an acequia in the east mountain area, Bernalillo County, New Mexico, 1995 .

2. Water-quality and ground-water-level data for wells in the northeast area, Bernalillo County, New Mexico, 1995 


\section{CONVERSION FACTORS AND VERTICAL DATUM}

\section{Multiply}

foot

mile

square mile
By

0.3048

1.609

2.590
To obtain

meter

kilometer

square kilometer

Temperature in degrees Celsius $\left({ }^{\circ} \mathrm{C}\right)$ can be converted to degrees Fahrenheit $\left({ }^{\circ} \mathrm{F}\right)$ by the equation:

$$
{ }^{\circ} \mathrm{F}=9 / 5\left({ }^{\circ} \mathrm{C}\right)+32
$$

Sea level: In this report sea level refers to the National Geodetic Vertical Datum of 1929-a geodetic datum derived from a general adjustment of the first-order level nets of the United States and Canada, formerly called Sea Level Datum of 1929. 


\title{
WATER-QUALITY AND GROUND-WATER-LEVEL DATA, BERNALILLO COUNTY, CENTRAL NEW MEXICO, 1995
}

\author{
By Dale R. Rankin
}

\begin{abstract}
Water-quality and ground-water-level data were collected in two areas of eastern Bernalillo County in central New Mexico between March and July of 1995. Fifty-one wells, two springs, and the Ojo Grande Acequia in the east mountain area of Bernalillo County and nine wells in the northeast area of the city of Albuquerque were sampled. The water samples were analyzed for selected nutrient species; total organic carbon; major dissolved constituents; dissolved arsenic, boron, iron, and manganese; and methylene blue active substances. Analytical results were used to compute hardness, sodium adsorption ratio, and dissolved solids. Specific conductance, $\mathrm{pH}$, temperature, and alkalinity were measured in the field at the time of sample collection. Groundwater-level and well-depth measurements were made at the time of sample collection when possible. Water-quality data, ground-water-level data, and well-depth data are presented in tabular form.
\end{abstract}

\section{INTRODUCTION}

Unincorporated areas of eastern Bernalillo County have undergone rapid development and a subsequent rise in population in recent years. As a result, the demand for ground-water supplies is greater and the potential for contamination has increased. The majority of homes in the unincorporated areas use septic systems for the disposal of wastewater. Bernalillo County officials recognize the importance of continuing and expanding monitoring efforts in these rapidly developing areas, with particular attention to the degradation of ground water that results from sewage disposal.

Accordingly, the Bernalillo County Environmental Health Department has entered into several cooperative agreements with the U.S. Geological Survey (USGS) since 1989 to collect ground-water data in the eastern part of Bernalillo County (fig. 1). In January 1990, the USGS began monitoring ground-water quality and ground-water levels in 20 domestic-supply wells in eastern Bernalillo County, referred to hereafter as the east mountain area. Water-quality samples were collected and ground-water-levels were measured monthly in the east mountain area between January 1990 and June 1993. Eleven domestic-supply wells in an unincorporated area northeast of the city of Albuquerque, referred to hereafter as the northeast area, were sampled once between December 1992 and September 1993 (Kues and Garcia, 1995). This report was prepared in cooperation with the Bernalillo County Environmental Health Department. 


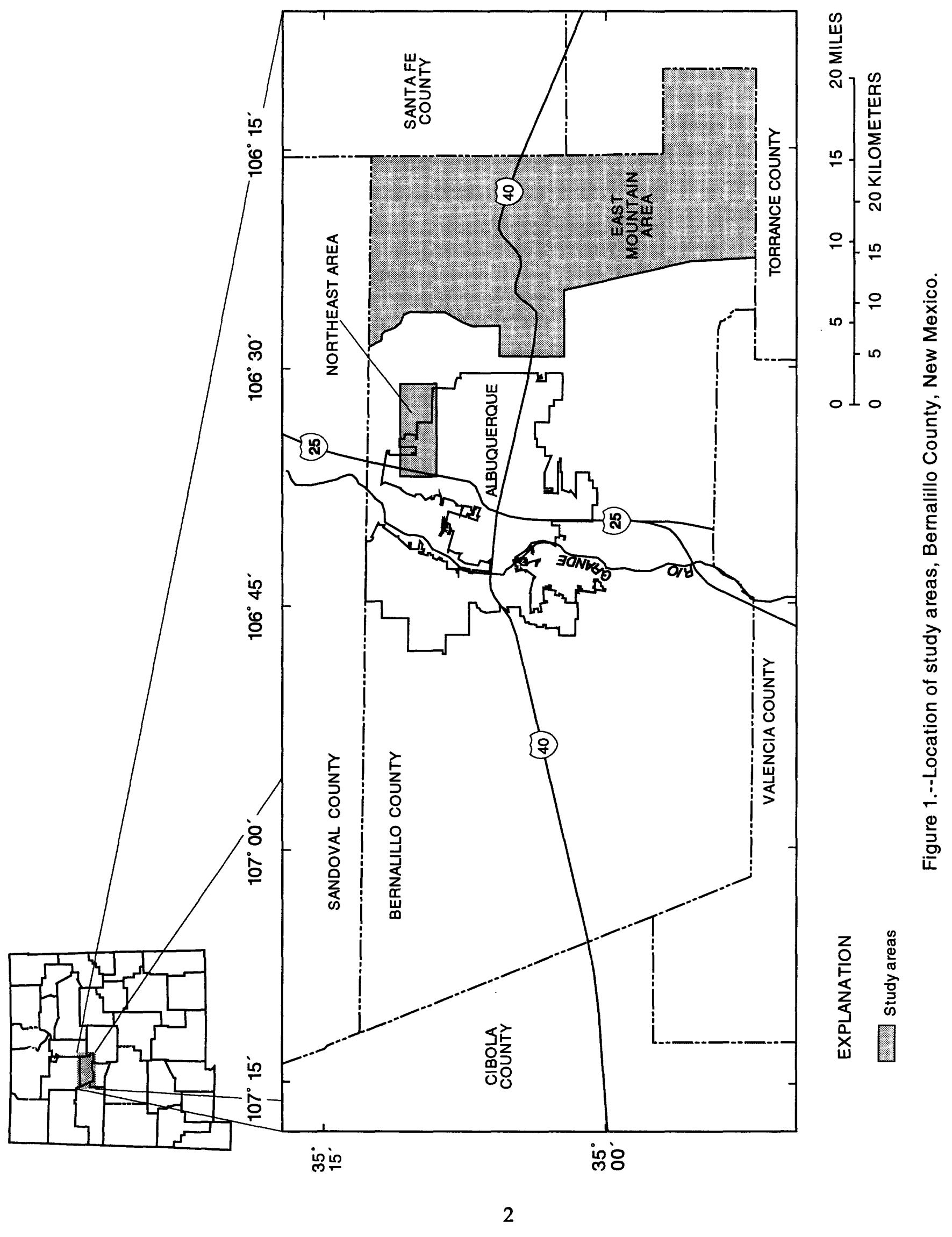




\section{Purpose and Scope}

This report presents water-quality data, ground-water-level data, and well-depth data collected in two unincorporated areas of eastern Bernalillo County between March and July of 1995. Water-quality data for 51 domestic-supply wells, 2 springs (sites 44 and 45), and the Ojo Grande Acequia (site 38) in the east mountain area and for 9 domestic-supply wells in the northeast area are presented in tabular form. Ground-water-level data and well-depth data are reported where available. Twenty of 51 wells in the east mountain area and 9 of 11 wells in the northeast area were sampled previously by the USGS (Kues and Garcia, 1995).

\section{Description of Study Areas}

The east mountain area comprises approximately 150 square miles in the eastern quarter of Bernalillo County. The boundaries of the area are the county lines on the north, east, and south, the crest line of the Sandia Mountains on the northwest, and the Manzano Mountains on the southwest (fig. 2). Soils and unconsolidated alluvial deposits overlie shallow, fractured limestone in most of the study area. Fractured sandstones and shales underlie the center part of the area, and igneous and metamorphic rock crop out on the south slopes of the Sandia Mountains and north slopes of the Manzano Mountains. The ground-water system and geology of the area were described by Titus (1980) and Kues (1990).

The northeast area (fig. 3) is underlain by unconsolidated alluvium and basin-fill deposits of clay, silt, sand, and gravel. The deposits range from 2,000 to 3,500 feet thick along the western flanks of the Sandia Mountains. The geohydrologic framework and hydrologic conditions of the Albuquerque Basin were summarized by Thorn and others (1993).

\section{Acknowledgments}

The cooperation of the well owners, whose kind permission allowed sampling and ground-water-level data collection to be conducted, is gratefully acknowledged. Appreciation is also extended to Bernalillo County Environmental Health Department personnel, particularly Mr. Jeffery Peterson; to State Engineer Office personnel, who furnished information on wells that were sampled; and to Mr. Chris Jinzo, Mayordomo of the Ojo Grande Acequia, who provided access to and information regarding the spring and acequia samples. 


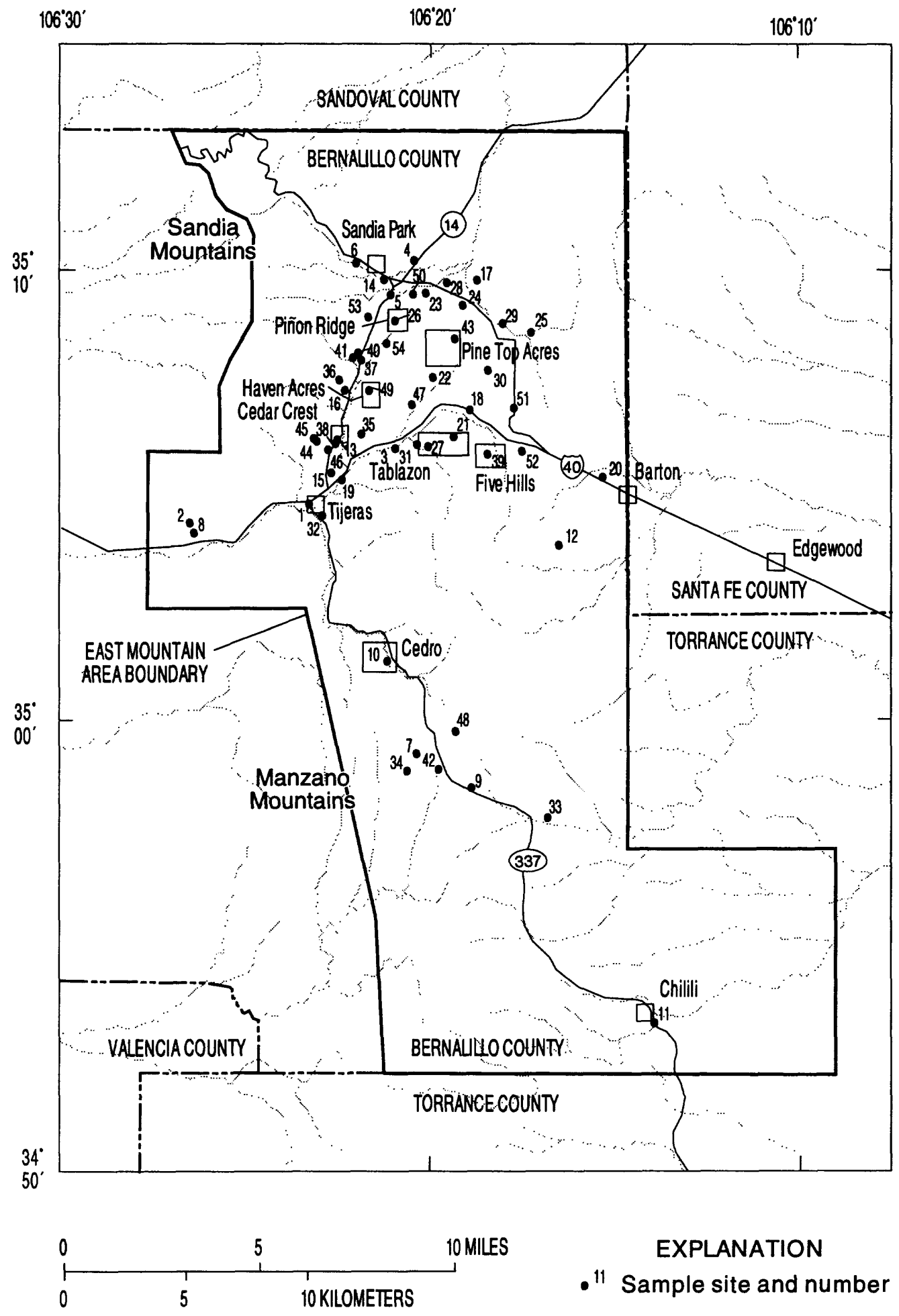

Figure 2.--Location of wells, springs, and acequia sampled in the east mountain area, Bernalillo County, New Mexico. 


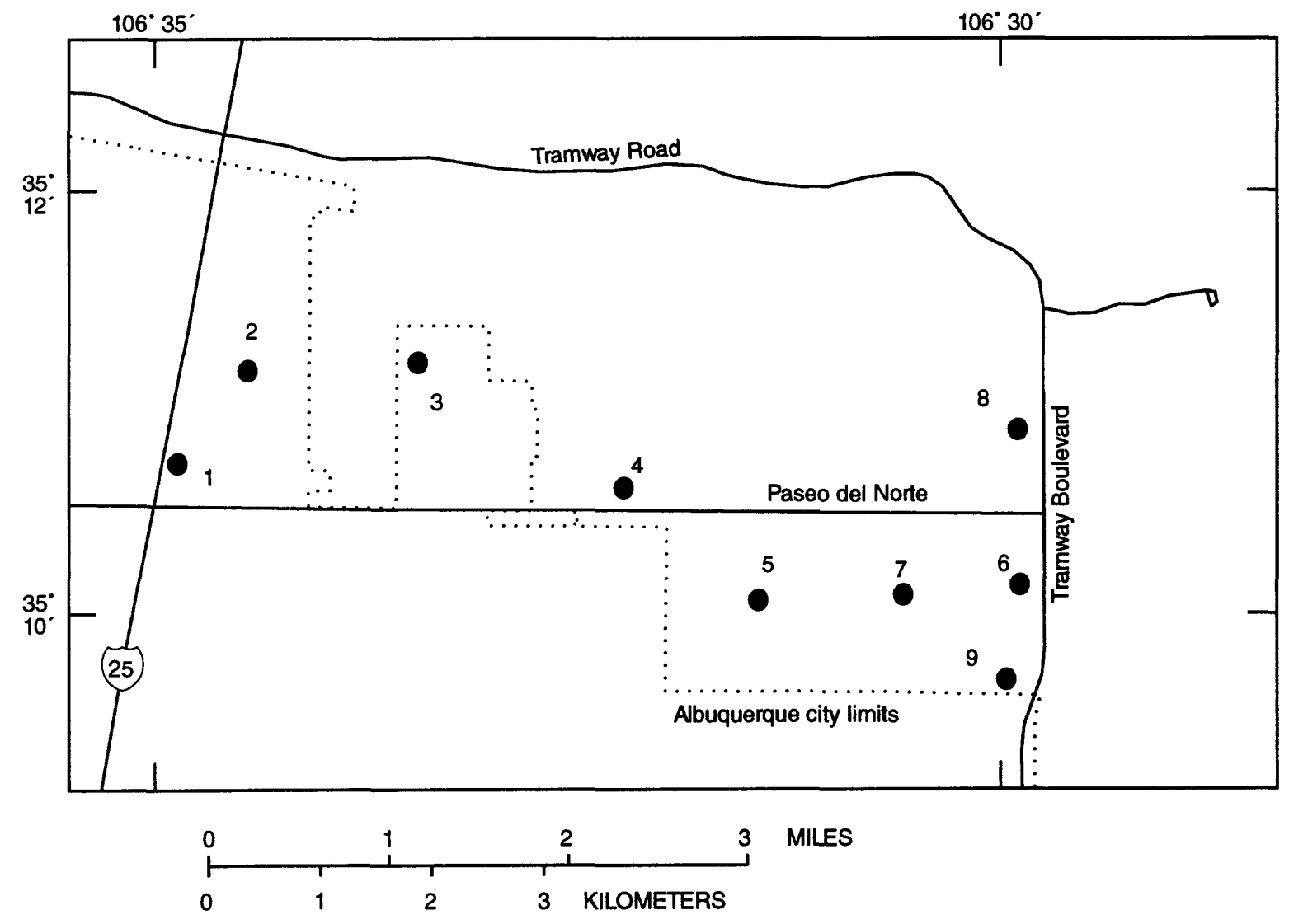

EXPLANATION

6

Sampled well and number

Figure 3.--Location of wells sampled in the northeast area, Bemalillo County, New Mexico. 


\section{METHODS}

Ground-water samples and water-quality field measurements were collected using standard USGS techniques described by Wood (1976). Water samples were obtained using submersible pumps installed in the domestic-supply wells. Samples were collected as close as possible to the well head and in all cases were collected prior to water treatment. For all samples collected from both areas, specific conductance, $\mathrm{pH}$, air and water temperature, and alkalinity were measured in the field at the time of sample collection. Samples were processed in the field and shipped the same day for analysis to the USGS National Water Quality Laboratory in Arvada, Colorado. Samples were analyzed for all constituents using standard USGS techniques described by Fishman and Friedman (1989). Prior to sample collection, ground-water levels were measured using a steel tape. In each case, a check measurement was made. Water-quality field measurements, including specific conductance, $\mathrm{pH}$, air and water temperature, and alkalinity, and analytical results were entered into the USGS National Water Information System (NWIS) water-quality data base (QWDATA); ground-water levels, well-depth measurements, and landsurface altitudes were entered into the NWIS Ground-Water Site-Inventory data base (GWSI).

\section{DISCUSSION OF DATA}

All water samples collected from the east mountain area and northeast area were analyzed for nutrients, including nitrate, nitrite, phosphorus, orthophosphate, and ammonia, which are common indicators of ground-water contamination from sewage effluent. Additionally, the samples were analyzed for other possible indicators of ground-water contamination from sewage effluent, including total organic carbon, dissolved boron, dissolved iron, and methylene blue active substances, which are detergent additives that were used until about the mid-1970's. The samples were also analyzed for major dissolved constituents, including calcium, magnesium, sodium, potassium, chloride, sulfate, and fluoride, and for dissolved silica, manganese, and arsenic. Total hardness, sodium adsorption ratio, and dissolved solids were computed from analytical results. Water-quality data for 51 wells, 2 springs, and the Ojo Grande Acequia in the east mountain area are presented in table 1 . Water-quality data for nine wells in the northeast area are presented in table 2 . In addition to the results of chemical analyses, both tables include site number, station identification number, sample collection date, and landsurface altitude of each well; when possible, a ground-water level (in feet below land surface) and well depth are included.

Nitrate concentrations are determined by analyzing the sample for the sum of nitrite plus nitrate and for nitrite separately, then calculating the concentration of nitrate by the difference. The sum concentrations of nitrite plus nitrate as nitrogen and the concentration of nitrite are displayed in tables 1 and 2, rather than the concentration of nitrite and nitrate separately, because the concentration of nitrite in most cases was less than the minimum reporting level (the minimum concentration of an analyte that can be reliably measured and reported by the laboratory) for the analytical method ( 0.01 milligram per liter $(\mathrm{mg} / \mathrm{L})$ as nitrogen); therefore, the concentration of nitrate could not be calculated. Because the concentration of nitrite in most samples was less than or near the minimum reporting level, the concentration of nitrate alone is approximately the same as that of nitrite plus nitrate. 
Water quality differs appreciably among the 51 wells sampled in the east mountain area. This is evident, for example, by the ranges of $\mathrm{pH}$ (from 6.4 in well 49 to 9.3 in well 16), specific conductance (from 385 microsiemens per centimeter at 25 degrees Celsius $(\mu \mathrm{S} / \mathrm{cm}$ ) in well 35 to $2,960 \mu \mathrm{S} / \mathrm{cm}$ in well 47 ), dissolved solids (from $229 \mathrm{mg} / \mathrm{L}$ in well 38 to $3,210 \mathrm{mg} / \mathrm{L}$ in well 35), and nitrite plus nitrate (from less than $0.050 \mathrm{mg} / \mathrm{L}$ in wells $15,22,43,47,49$, and 54 to $21.0 \mathrm{mg} / \mathrm{L}$ in well 5); other constituents display similar variability. Water from well 5 had the largest concentration of nitrite plus nitrate in 1995, and the concentration of this constituent is similar to concentrations reported for this well by Kues and Garcia (1995).

In the northeast area, the largest concentrations of dissolved nitrite plus nitrate were from well $8(2.80 \mathrm{mg} / \mathrm{L})$ and well $9(2.40 \mathrm{mg} / \mathrm{L})$ located along Tramway Road (fig. 3) on the eastern edge of the study area. Concentrations of dissolved nitrite plus nitrate in water from the seven other northeast area wells were less than or equal to $0.28 \mathrm{mg} / \mathrm{L}$. Results from this study are similar to results obtained in 1992-93 (Kues and Garcia, 1995, table 2). The nitrite plus nitrate concentration in well 1 exhibited the most change, increasing from $0.051 \mathrm{mg} / \mathrm{L}$ in 1993 to 0.170 $\mathrm{mg} / \mathrm{L}$ in 1995. 


\section{REFERENCES CITED}

Fishman, M.J., and Friedman, L.C., eds., 1989, Methods for the determination of inorganic substances in water and fluvial sediments: U.S. Geological Survey Techniques of WaterResources Investigations, book 5, chap. A1, 545 p.

Kues, G.E., 1990, Ground-water availability and quality in eastern Bernalillo County and vicinity, central New Mexico: U.S. Geological Survey Water-Resources Investigations Report 894127,82 p.

Kues, G.E., and Garcia, B.M., 1995, Ground-water quality and ground-water-level data, Bernalillo County, central New Mexico, 1990-93: U.S. Geological Survey Open-File Report 95-385, $76 \mathrm{p}$.

Thorn, C.R., McAda, D.P., and Kernodle, J.M., 1993, Geohydrologic framework and hydrologic conditions in the Albuquerque Basin, central New Mexico: U.S. Geological Survey WaterResources Investigations Report 93-4149, 106 p.

Titus, F.B., 1980, Ground water in the Sandia and northern Manzano Mountains, New Mexico: Socorro, New Mexico Bureau of Mines and Mineral Resources Hydrologic Report 6, 66 p.

Wood, W.W., 1976, Guidelines for collection and field analysis of ground-water samples for selected unstable constituents: U.S. Geological Survey Techniques of Water-Resources Investigations, book 1, chap. D2, 24 p. 


\section{Table 1.--Water-quality and ground-water-level data for wells, springs, and an acequia in the east mountain area, Bernalillo County, New Mexico, 1995}

[Analyses are for dissolved constituents unless otherwise noted; $\mu \mathrm{S} / \mathrm{cm}$, microsiemens per centimeter at 25 degrees Celsius; deg C, degrees Celsius; $\mathrm{mg} / \mathrm{L}$, milligrams per liter; $\mu \mathrm{g} / \mathrm{L}$, micrograms per liter; <, less than; -, no data; NA, not applicable; depth to water: A, artesian well]

\begin{tabular}{|c|c|c|c|c|c|c|c|c|c|c|c|c|}
\hline $\begin{array}{c}\text { site } \\
\text { number } \\
\text { (figure } \\
\text { 2) }\end{array}$ & $\begin{array}{c}\text { Station } \\
\text { identification } \\
\text { number }\end{array}$ & $\begin{array}{c}\text { Date } \\
\text { sampled }\end{array}$ & $\begin{array}{c}\text { Ni- } \\
\text { trite } \\
\text { plus } \\
\text { nitrate } \\
\text { (mg/L } \\
\text { as N) }\end{array}$ & $\begin{array}{c}\text { Ni- } \\
\text { trite } \\
\text { (mg/L } \\
\text { as N) }\end{array}$ & $\begin{array}{l}\text { Phos- } \\
\text { phorus } \\
\text { (mg/L } \\
\text { as P) }\end{array}$ & $\begin{array}{l}\text { Ortho- } \\
\text { phos- } \\
\text { phate } \\
\text { (mg/L } \\
\text { as P) }\end{array}$ & $\begin{array}{c}\text { Ammo- } \\
\text { nla } \\
(\mathrm{mg} / \mathrm{L} \\
\text { as N) }\end{array}$ & $\begin{array}{c}\text { Ammonia } \\
\text { plus } \\
\text { organic } \\
\text { ni- } \\
\text { trogen } \\
\text { (mg/L } \\
\text { as } N \text { ) }\end{array}$ & $\begin{array}{l}\text { Total } \\
\text { organic } \\
\text { carbon } \\
\text { (mg/L) }\end{array}$ & $\begin{array}{l}\text { Boron } \\
\text { (mg/L) }\end{array}$ & $\begin{array}{c}\text { Iron } \\
\text { (mg/L) }\end{array}$ & $\begin{array}{c}\text { Methy- } \\
\text { lene } \\
\text { blue } \\
\text { act ive } \\
\text { sub- } \\
\text { stances } \\
\text { (mg/L) }\end{array}$ \\
\hline 1 & 350449106231901 & $03-14-95$ & 0.660 & 0.010 & $<0.010$ & $<0.010$ & $<0.015$ & $<0.20$ & 3.3 & 190 & 100 & $<0.02$ \\
\hline 2 & 350423106263301 & $03-17-95$ & 0.720 & $<0.010$ & $<0.010$ & $<0.010$ & $<0.015$ & $<0.20$ & 4.9 & 30 & 8 & $<0.02$ \\
\hline 3 & 350604106205801 & $03-22-95$ & 9.20 & $<0.010$ & 0.010 & 0.020 & 0.020 & $<0.20$ & 12 & 110 & 7 & $<0.02$ \\
\hline 4 & 351014106202801 & $03-23-95$ & 2.70 & $<0.010$ & 0.010 & 0.010 & $<0.015$ & $<0.20$ & 6.0 & 50 & $<3$ & $<0.02$ \\
\hline 5 & 350930106210701 & $03-23-95$ & 21.0 & $<0.010$ & 0.020 & 0.020 & $<0.015$ & $<0.20$ & 4.2 & 60 & $<3$ & $<0.02$ \\
\hline 6 & 351011106220401 & $03-24-95$ & 0.130 & $<0.010$ & $<0.010$ & $<0.010$ & $<0.015$ & $<0.20$ & 4.5 & 20 & $<3$ & $<0.02$ \\
\hline 7 & 345918106202001 & $03-28-95$ & 0.050 & $<0.010$ & $<0.010$ & $<0.010$ & $<0.015$ & $<0.20$ & 3.4 & 280 & 13 & $<0.02$ \\
\hline 8 & 350410106262601 & $03-28-95$ & 14.0 & $<0.010$ & $<0.010$ & $<0.010$ & $<0.015$ & $<0.20$ & 1.0 & 50 & 5 & $<0.02$ \\
\hline 9 & 345833106185101 & $03-28-95$ & 1.30 & $<0.010$ & 0.020 & 0.030 & $<0.015$ & 0.20 & 9.9 & 120 & 29 & 0.02 \\
\hline 10 & 350119106210901 & $03-29-95$ & 4.50 & $<0.010$ & 0.010 & 0.020 & $<0.015$ & $<0.20$ & 5.6 & 40 & 7 & $<0.02$ \\
\hline 11 & 345319106135101 & $03-31-95$ & 3.10 & $<0.010$ & 0.050 & 0.030 & $<0.015$ & $<0.20$ & 2.7 & 60 & 6 & 0.04 \\
\hline 12 & 350356106162901 & $03-31-95$ & 0.080 & $<0.010$ & $<0.010$ & $<0.010$ & 0.030 & $<0.20$ & 12 & 320 & 14 & 0.03 \\
\hline 13 & 350615106223301 & $04-05-95$ & 7.20 & 0.020 & 0.020 & 0.020 & $<0.015$ & $<0.20$ & 0.8 & 50 & 4 & $<0.02$ \\
\hline 14 & 350949106211801 & $04-12-95$ & 0.630 & $<0.010$ & $<0.010$ & $<0.010$ & $<0.015$ & $<0.20$ & 3.4 & 20 & $<3$ & $<0.02$ \\
\hline 15 & 350531106224301 & $04-12-95$ & $<0.050$ & $<0.010$ & $<0.010$ & $<0.010$ & $<0.015$ & $<0.20$ & 11 & 50 & 19 & $<0.02$ \\
\hline 16 & 350721106222101 & $04-13-95$ & 0.330 & 0.020 & 0.030 & 0.020 & $<0.015$ & $<0.20$ & 1.5 & 10 & 9 & $<0.02$ \\
\hline 17 & 350949106184501 & $04-14-95$ & 1.20 & $<0.010$ & $<0.010$ & $<0.010$ & $<0.015$ & $<0.20$ & 3.9 & 70 & 7 & $<0.02$ \\
\hline 18 & 350655106185601 & $04-24-95$ & 9.40 & $<0.010$ & $<0.010$ & $<0.010$ & $<0.015$ & 0.20 & 12 & 50 & 5 & 0.04 \\
\hline 19 & 350522106222501 & $04-24-95$ & 2.60 & $<0.010$ & $<0.010$ & 0.010 & $<0.015$ & $<0.20$ & 8.9 & 50 & 5 & $<0.02$ \\
\hline 20 & 350525106151701 & $04-25-95$ & 7.60 & $<0.010$ & $<0.010$ & 0.010 & $<0.015$ & 0.20 & 12 & 50 & 10 & 0.02 \\
\hline 21 & 350620106192201 & $04-25-95$ & 0.560 & $<0.010$ & $<0.010$ & $<0.010$ & $<0.015$ & $<0.20$ & 4.2 & 150 & 5 & $<0.02$ \\
\hline 22 & 350739106195601 & $04-25-95$ & $<0.050$ & $<0.010$ & $<0.010$ & $<0.010$ & 0.210 & 0.30 & 8.5 & 180 & 360 & 0.02 \\
\hline 23 & 350931106200901 & $04-26-95$ & 2.00 & $<0.010$ & 0.030 & 0.020 & 0.020 & $<0.20$ & 1.8 & 30 & 5 & $<0.02$ \\
\hline 24 & 350908106190601 & $04-26-95$ & 0.180 & $<0.010$ & 0.020 & 0.010 & $<0.015$ & $<0.20$ & 5.2 & 70 & 15 & $<0.02$ \\
\hline 25 & 350840106171601 & $04-26-95$ & 5.80 & $<0.010$ & 0.030 & 0.030 & $<0.015$ & $<0.20$ & 2.4 & 50 & $<3$ & $<0.02$ \\
\hline 26 & 350854106210001 & $04-28-95$ & 5.20 & $<0.010$ & $<0.010$ & $<0.010$ & $<0.015$ & $<0.20$ & 8.6 & 80 & $<3$ & 0.04 \\
\hline 27 & 350606106200401 & $05-30-95$ & 0.480 & $<0.010$ & $<0.010$ & $<0.010$ & $<0.015$ & 2.1 & 1.5 & 60 & $<3$ & $<0.02$ \\
\hline 28 & 350946106193501 & $05-31-95$ & 3.20 & $<0.010$ & $<0.010$ & $<0.010$ & 0.020 & $<0.20$ & 0.9 & 150 & $<3$ & 0.02 \\
\hline 29 & 350851106180301 & $05-31-95$ & 4.20 & $<0.010$ & $<0.010$ & 0.020 & 0.020 & 0.20 & 0.5 & 40 & $<3$ & $<0.02$ \\
\hline 30 & 350748106182601 & $05-31-95$ & 4.40 & $<0.010$ & $<0.010$ & $<0.010$ & 0.020 & $<0.20$ & 1.5 & 70 & $<3$ & $<0.02$ \\
\hline 31 & 350609106202201 & $06-01-95$ & 1.70 & 0.020 & $<0.010$ & 0.010 & 0.020 & $<0.20$ & 2.6 & 230 & $<3$ & $<0.02$ \\
\hline 32 & 350434106225701 & $06-01-95$ & 1.20 & $<0.010$ & $<0.010$ & $<0.010$ & $<0.015$ & $<0.20$ & 0.4 & 70 & 10 & 0.04 \\
\hline 33 & 345754106164601 & $06-02-95$ & 3.70 & $<0.010$ & $<0.010$ & $<0.010$ & 0.020 & $<0.20$ & 2.3 & 70 & $<3$ & 0.04 \\
\hline 34 & 345855106203601 & $06-02-95$ & 4.70 & $<0.010$ & $<0.010$ & 0.020 & 0.020 & $<0.20$ & 2.4 & 50 & $<3$ & 0.03 \\
\hline 35 & 350623106215301 & $06-02-95$ & 1.20 & $<0.010$ & $<0.010$ & $<0.010$ & 0.080 & $<0.20$ & 3.1 & 140 & 70 & $<0.02$ \\
\hline 36 & 350735106223001 & $06-06-95$ & 0.320 & $<0.010$ & $<0.010$ & $<0.010$ & $<0.015$ & $<0.20$ & 1.0 & 250 & 3 & 0.07 \\
\hline 37 & 350802106215401 & $06-06-95$ & 7.00 & 0.030 & $<0.010$ & $<0.010$ & $<0.015$ & $<0.20$ & 2.2 & 230 & 16 & 0.03 \\
\hline $1_{38}$ & 350610106223501 & $06-06-95$ & 0.140 & $<0.010$ & $<0.010$ & $<0.010$ & 0.020 & $<0.20$ & 3.2 & 20 & $<3$ & 0.04 \\
\hline 39 & 350557106182701 & $06-07-95$ & 7.20 & 0.010 & & 0.010 & 0.030 & $<0.20$ & 1. & 50 & 4 & 0.05 \\
\hline 40 & 350812106220001 & $06-07-95$ & 3.30 & $<0.010$ & 0.050 & 0.040 & 0.030 & $<0.20$ & 1.1 & 90 & $<3$ & 0.02 \\
\hline 41 & 350805106220801 & $06-07-95$ & 7.90 & $<0.010$ & 0.010 & 0.020 & 0.030 & $<0.20$ & 1.5 & 100 & $<3$ & $<0.02$ \\
\hline 42 & 345858106194601 & $06-08-95$ & 8.10 & $<0.010$ & $<0.010$ & 0.040 & 0.030 & $<0.20$ & 3.3 & 50 & 8 & $<0.02$ \\
\hline 43 & 350831106192301 & $06-08-95$ & $<0.050$ & $<0.010$ & $<0.010$ & $<0.010$ & 1.10 & 0.90 & 0.9 & 230 & 2,100 & $<0.02$ \\
\hline 244 & 350617106231101 & $06-08-95$ & 0.180 & $<0.010$ & $<0.010$ & $<0.010$ & 0.020 & $<0.20$ & 0.7 & $<10$ & $<3$ & 0.02 \\
\hline $\begin{array}{r}245 \\
46\end{array}$ & $\begin{array}{l}350613106230601 \\
350602106224701\end{array}$ & $\begin{array}{l}06-08-95 \\
06-09-95\end{array}$ & $\begin{array}{l}0.170 \\
5.10\end{array}$ & $\begin{array}{r}<0.010 \\
0.020\end{array}$ & $\begin{array}{l}<0.010 \\
<0.010\end{array}$ & $\begin{array}{l}<0.010 \\
<0.010\end{array}$ & $\begin{array}{l}0.020 \\
0.020\end{array}$ & $\begin{array}{l}<0.20 \\
<0.20\end{array}$ & $\begin{array}{l}1.3 \\
1.0\end{array}$ & $\begin{array}{l}20 \\
60\end{array}$ & $\begin{array}{l}<3 \\
<3\end{array}$ & $\begin{array}{r}<0.02 \\
0.03\end{array}$ \\
\hline 47 & 350702106203101 & $06-12-95$ & $<0.050$ & $<0.010$ & $<0.010$ & $<0.010$ & 0.380 & 0.50 & 1.9 & 260 & 700 & 0.04 \\
\hline 48 & 345948106191701 & $06-12-95$ & 0.560 & $<0.010$ & 0.020 & 0.010 & 0.020 & $<0.20$ & 1.3 & 60 & 4 & $<0.02$ \\
\hline 49 & 350721106214101 & $06-14-95$ & $<0.050$ & $<0.010$ & 0.020 & $<0.010$ & 0.100 & $<0.20$ & 1.2 & 70 & $<10$ & $<0.02$ \\
\hline 50 & 350930106203001 & $06-14-95$ & 1.90 & $<0.010$ & 0.050 & 0.010 & 0.020 & $<0.20$ & 1.1 & 20 & $<3$ & $<0.02$ \\
\hline 51 & 350658106174401 & $06-14-95$ & 0.790 & 0.010 & & $<0.010$ & 0.030 & & 2.7 & 100 & $<10$ & \\
\hline 52 & 350601106173101 & $07-25-95$ & 0.100 & $<0.010$ & $<0.010$ & $<0.010$ & 0.030 & $<0.20$ & 3.3 & 130 & 70 & $<0.02$ \\
\hline 53 & 350859106214301 & $07-26-95$ & 9.70 & $<0.010$ & 0.040 & 0.020 & $<0.015$ & $<0.20$ & 3.7 & 120 & $<3$ & $<0.02$ \\
\hline 54 & 350825106211101 & $07-27-95$ & $<0.050$ & $<0.010$ & $<0.010$ & 0.010 & 0.430 & 0.50 & 0.9 & 480 & 140 & $<0.02$ \\
\hline
\end{tabular}


Table 1.--Water-quality and ground-water-level data for wells, springs, and an acequia in the east mountain area, Bernalillo County, New Mexico, 1995-Continued

\begin{tabular}{|c|c|c|c|c|c|c|c|c|c|c|c|}
\hline $\begin{array}{l}\text { site } \\
\text { number } \\
\text { (f1gure } \\
\text { 2) }\end{array}$ & $\begin{array}{c}\text { Station } \\
\text { 1dent } 1 \text { fication } \\
\text { number }\end{array}$ & $\begin{array}{c}\text { Date } \\
\text { sampled }\end{array}$ & $\begin{array}{c}\text { Calcium } \\
(\mathrm{mg} / \mathrm{L})\end{array}$ & $\begin{array}{l}\text { Magne- } \\
\text { s1um } \\
(\mathrm{mg} / \mathrm{L})\end{array}$ & $\begin{array}{l}\text { sod1 um } \\
\text { (mg/L) }\end{array}$ & $\begin{array}{l}\text { Potas- } \\
\text { sium } \\
(\mathrm{mg} / \mathrm{L})\end{array}$ & $\begin{array}{l}\text { Chlo- } \\
\text { ride } \\
(\mathrm{mg} / \mathrm{I})\end{array}$ & $\begin{array}{c}\text { Sulfate } \\
\text { (mg/L) }\end{array}$ & $\begin{array}{l}\text { Fluo- } \\
\text { ride } \\
\text { (mg/L) }\end{array}$ & $\begin{array}{l}\mathrm{s} 111 \mathrm{ca} \\
(\mathrm{mg} / \mathrm{L})\end{array}$ & $\begin{array}{c}\text { Manga- } \\
\text { nese } \\
(\mathrm{mg} / \mathrm{L})\end{array}$ \\
\hline $\begin{array}{l}1 \\
2\end{array}$ & $\begin{array}{l}350449106231901 \\
350423106263301\end{array}$ & $\begin{array}{l}03-14-95 \\
03-17-95\end{array}$ & $\begin{array}{l}74 \\
73\end{array}$ & $\begin{array}{l}46 \\
18\end{array}$ & $\begin{array}{l}56 \\
24\end{array}$ & $\begin{array}{l}5.3 \\
3.7\end{array}$ & $\begin{array}{r}130 \\
8.9\end{array}$ & $\begin{array}{l}88 \\
74\end{array}$ & $\begin{array}{l}0.20 \\
2.1\end{array}$ & $\begin{array}{l}17 \\
19\end{array}$ & $\begin{array}{l}19 \\
<1\end{array}$ \\
\hline 3 & 350604106205801 & $03-22-95$ & 180 & 45 & 71 & 2.9 & 310 & 67 & 0.20 & 20 & $<1$ \\
\hline 4 & 351014106202801 & $03-23-95$ & 110 & 21 & 16 & 1.3 & 120 & 39 & 0.30 & 26 & $<1$ \\
\hline 6 & 351011106220401 & $03-24-95$ & 130 & 13 & 30 & 1.1 & 80 & 34 & 0.60 & 17 & $<1$ \\
\hline 7 & 345918106202001 & $03-28-95$ & 8.7 & 7.3 & 160 & 4.1 & 34 & 21 & 4.9 & 10 & $<1$ \\
\hline 8 & 350410106262601 & $03-28-95$ & 190 & 37 & 48 & 4.0 & 180 & 160 & 1.8 & 21 & 1 \\
\hline 9 & 345833106185101 & $03-28-95$ & 160 & 32 & 140 & 3.7 & 160 & 170 & 1.1 & 20 & 8 \\
\hline 10 & 350119106210901 & $03-29-95$ & 160 & 29 & 47 & 1.9 & 140 & 51 & 0.40 & 15 & 3 \\
\hline 11 & 345319106135101 & $03-31-95$ & 130 & 15 & 41 & 2.1 & 31 & 49 & 0.20 & 18 & $<1$ \\
\hline 12 & 350356106162901 & $03-31-95$ & 27 & 22 & 170 & 4.8 & 55 & 83 & 4.8 & 11 & 6 \\
\hline 13 & 350615106223301 & $04-05-95$ & 140 & 26 & 30 & 0.80 & 19 & 100 & 0.20 & 20 & 1 \\
\hline 15 & 350531106224301 & $04-12-95$ & 200 & 34 & 39 & 1.9 & 97 & 270 & 0.30 & 19 & 4 \\
\hline 16 & 350721106222101 & $04-13-95$ & 1.1 & 0.0 & 170 & 0.10 & 14 & 40 & 0.90 & 10 & 4 \\
\hline 17 & 350949106184501 & $04-14-95$ & 83 & 29 & 23 & 1.3 & 23 & 56 & 0.30 & 27 & 2 \\
\hline 18 & 350655106185601 & $04-24-95$ & 200 & 52 & 63 & 4.1 & 290 & 150 & 0.20 & 16 & $<1$ \\
\hline 19 & 350522106222501 & $04-24-95$ & 190 & 36 & 77 & 2.1 & 190 & 240 & 0.20 & 20 & $<1$ \\
\hline 20 & 350525106151701 & $04-25-95$ & 260 & 42 & 57 & 1.8 & 450 & 130 & 0.30 & 19 & $<10$ \\
\hline 21 & 350620106192201 & $04-25-95$ & 50 & 24 & 84 & 5.5 & 45 & 69 & 1.2 & 10 & 2 \\
\hline 22 & 350739106195601 & $04-25-95$ & 160 & 66 & 60 & 5.3 & 35 & 290 & 0.40 & 23 & 18 \\
\hline 23 & 350931106200901 & $04-26-95$ & 310 & 17 & 19 & 1.8 & 20 & 660 & 0.30 & 23 & $<1$ \\
\hline 24 & 350908106190601 & $04-26-95$ & 120 & 21 & 22 & 2.1 & 3.9 & 210 & 0.30 & 21 & 2 \\
\hline 25 & 350840106171601 & $04-26-95$ & 140 & 20 & 21 & 1.9 & 140 & 100 & 0.30 & 18 & $<1$ \\
\hline 26 & 350854106210001 & $04-28-95$ & 130 & 41 & 26 & 3.8 & 97 & 94 & 0.30 & 22 & 6 \\
\hline 27 & 350606106200401 & $05-30-95$ & 84 & 19 & 22 & 1.8 & 21 & 66 & 0.40 & 16 & $<1$ \\
\hline 28 & 350946106193501 & $05-31-95$ & 52 & 26 & 47 & 2.1 & 51 & 80 & 0.90 & 23 & 2 \\
\hline 29 & 350851106180301 & $05-31-95$ & 92 & 15 & 14 & 1.7 & 20 & 25 & 0.30 & 19 & 3 \\
\hline 30 & 350748106182601 & $05-31-95$ & 120 & 35 & 33 & 2.2 & 200 & 68 & 0.30 & 18 & $<1$ \\
\hline 34 & 345855106203601 & $06-02-95$ & 150 & 17 & 32 & 1.5 & 82 & 76 & 0.20 & 19 & $<1$ \\
\hline 35 & 350623106215301 & $06-02-95$ & 400 & 200 & 310 & 50 & 180 & 1,800 & 0.50 & 19 & 30 \\
\hline 36 & 350735106223001 & $06-06-95$ & 77 & 20 & 52 & 0.90 & 43 & 42 & 0.30 & 16 & $<1$ \\
\hline 37 & 350802106215401 & $06-06-95$ & 210 & 43 & 40 & 2.6 & 210 & 250 & 0.20 & 19 & 2 \\
\hline $1_{38}$ & 350610106223501 & $06-06-95$ & 70 & 9.6 & 6.7 & 0.70 & 4.0 & 15 & 0.20 & 18 & $<1$ \\
\hline 39 & 350557106182701 & $06-07-95$ & 190 & 45 & 42 & 2.5 & 210 & 200 & 0.40 & 14 & 5 \\
\hline 40 & 350812106220001 & $06-07-95$ & 110 & 15 & 26 & 0.80 & 53 & 28 & 0.30 & 25 & $<1$ \\
\hline 41 & 350805106220801 & $06-07-95$ & 120 & 21 & 31 & 0.70 & 77 & 45 & 0.30 & 24 & $<1$ \\
\hline 42 & 345858106194601 & $06-08-95$ & 190 & 13 & 26 & 0.90 & 140 & 43 & 0.20 & 19 & $<1$ \\
\hline 43 & 350831106192301 & $06-08-95$ & 60 & 55 & 190 & 4.4 & 17 & 270 & 0.30 & 18 & 12 \\
\hline 244 & 350617106231101 & $06-08-95$ & 84 & 9.5 & 6.6 & 0.70 & 4.0 & 14 & 0.20 & 17 & $<1$ \\
\hline${ }^{2} 45$ & 350613106230601 & $06-08-95$ & 87 & 9.6 & 6.6 & 0.70 & 3.9 & 14 & 0.20 & 17 & $<1$ \\
\hline 46 & 350602106224701 & $06-09-95$ & 100 & 19 & 55 & 2.0 & 12 & 98 & 0.20 & 16 & 4 \\
\hline 47 & 350702106203101 & $06-12-95$ & 280 & 150 & 230 & 7.4 & 190 & 1,000 & 0.40 & 18 & 60 \\
\hline 48 & 345948106191701 & $06-12-95$ & 120 & 34 & 22 & 3.7 & 45 & 63 & 0.30 & 13 & $<1$ \\
\hline 49 & 350721106214101 & $06-14-95$ & 430 & 140 & 45 & 4.2 & 50 & 1,200 & 0.30 & 24 & 140 \\
\hline 50 & 350930106203001 & $06-14-95$ & 86 & 4.4 & 12 & 0.70 & 20 & 10 & 0.20 & 18 & 2 \\
\hline 51 & 350658106174401 & $06-14-95$ & 220 & 85 & 150 & 3.3 & 520 & 220 & 0.40 & 16 & $<10$ \\
\hline 52 & 350601106173101 & $07-25-95$ & 310 & 140 & 73 & 5.0 & 470 & 640 & 0.30 & 19 & 30 \\
\hline 53 & 350859106214301 & $07-26-95$ & 180 & 31 & 24 & 1.5 & 220 & 29 & 0.20 & 27 & 12 \\
\hline 54 & 350825106211101 & $07-27-95$ & 3.0 & 1.7 & 290 & 1.9 & 6.5 & 150 & 0.60 & 9.6 & 5 \\
\hline
\end{tabular}


Table 1.--Water-quality and ground-water-level data for wells, springs, and an acequia in the east mountain area, Bernalillo County, New Mexico, 1995--Continued

\begin{tabular}{|c|c|c|c|c|c|c|c|c|c|c|c|}
\hline $\begin{array}{l}\text { site } \\
\text { number } \\
\text { (f1gure } \\
\text { 2) }\end{array}$ & $\begin{array}{c}\text { Station } \\
\text { 1dent } 1 \text { ficat ion } \\
\text { number }\end{array}$ & $\begin{array}{l}\text { Date } \\
\text { sampled }\end{array}$ & $\begin{array}{c}\text { Arsenic } \\
(\mu g / L)\end{array}$ & $\begin{array}{c}\text { Total } \\
\text { hard- } \\
\text { ness } \\
\text { (mg/I } \\
\text { as } \\
\left.\mathrm{CaCO}_{3}\right\rangle\end{array}$ & $\begin{array}{l}\text { Sod1um } \\
\text { adsorp- } \\
\text { t1on } \\
\text { rat1o }\end{array}$ & $\begin{array}{l}\text { D1s- } \\
\text { solved } \\
\text { solids } \\
\text { (mg/L) }\end{array}$ & $\begin{array}{l}\text { Spe- } \\
\text { c1f1c } \\
\text { con- } \\
\text { duct- } \\
\text { ance } \\
(\mu S / \mathrm{cm})\end{array}$ & $\underset{\text { (units) }}{\mathrm{pH}}$ & $\begin{array}{l}\text { Temper- } \\
\text { ature } \\
\text { air } \\
\text { (deg } C \text { ) }\end{array}$ & $\begin{array}{l}\text { Temper- } \\
\text { ature } \\
\text { water } \\
\text { (deg C) }\end{array}$ & $\begin{array}{c}\text { Alka- } \\
\text { linity } \\
\text { (mg/L } \\
\text { as } \\
\left.\mathrm{CaCO}_{3}\right)\end{array}$ \\
\hline $\begin{array}{l}1 \\
2\end{array}$ & $\begin{array}{l}350449106231901 \\
350423106263301\end{array}$ & $\begin{array}{l}03-14-95 \\
03-17-95\end{array}$ & $\begin{array}{r}2 \\
<1\end{array}$ & $\begin{array}{l}370 \\
260\end{array}$ & $\begin{array}{l}1 \\
0.7\end{array}$ & $\begin{array}{l}558 \\
353\end{array}$ & $\begin{array}{l}952 \\
577\end{array}$ & $\begin{array}{l}7.4 \\
7.3\end{array}$ & $\begin{array}{l}13.0 \\
24.0\end{array}$ & $\begin{array}{l}14.0 \\
17.0\end{array}$ & $\begin{array}{l}222 \\
208\end{array}$ \\
\hline 3 & 350604106205801 & $03-22-95$ & $<1$ & 630 & 1 & 908 & 1,620 & 7.1 & 15.0 & 13.5 & 278 \\
\hline 4 & 351014106202801 & $03-23-95$ & 2 & 360 & 0.4 & 452 & 825 & 7.2 & 11.5 & 12.0 & 174 \\
\hline 5 & 350930106210701 & $03-23-95$ & 1 & 560 & 0.6 & 738 & 1,270 & 7.0 & 11.0 & 13.0 & 298 \\
\hline 6 & 351011106220401 & $03-24-95$ & $<1$ & 380 & 0.7 & 488 & 856 & 6.9 & 10.0 & 9.0 & 300 \\
\hline 7 & 345918106202001 & $03-28-95$ & $<1$ & 52 & 10 & 445 & 784 & 7.9 & -0.5 & 11.0 & -- \\
\hline 8 & 350410106262601 & $03-28-95$ & $<1$ & 630 & 0.8 & 845 & 1,450 & 7.3 & 6.0 & 14.0 & -- \\
\hline $\begin{array}{r}9 \\
10\end{array}$ & $\begin{array}{l}345833106185101 \\
350119106210901\end{array}$ & $\begin{array}{l}03-28-95 \\
03-29-95\end{array}$ & $\begin{array}{l}28 \\
<1\end{array}$ & $\begin{array}{l}530 \\
520\end{array}$ & $\begin{array}{l}3 \\
0.9\end{array}$ & $\begin{array}{l}938 \\
674\end{array}$ & $\begin{array}{l}1,580 \\
1,220\end{array}$ & $\begin{array}{l}7.1 \\
7.1\end{array}$ & $\begin{array}{l}0.0 \\
0.0\end{array}$ & $\begin{array}{l}11.0 \\
10.0\end{array}$ & 354 \\
\hline 11 & 345319106135101 & $03-31-95$ & $<1$ & 390 & 0.9 & 527 & 890 & 7.1 & 8.0 & 11.0 & 376 \\
\hline 12 & 350356106162901 & $03-31-95$ & $<1$ & 160 & 6 & 602 & 1,040 & 7.6 & 9.0 & 15.5 & 372 \\
\hline 13 & 350615106223301 & $04-05-95$ & $<1$ & 460 & 0.6 & 582 & 935 & 7.3 & 15.5 & 12.0 & 348 \\
\hline 14 & 350949106211801 & $04-12-95$ & $<1$ & 310 & 0.3 & 380 & 666 & 7.0 & 9.0 & 7.0 & 247 \\
\hline 15 & 350531106224301 & $04-12-95$ & $<1$ & 640 & 0.7 & 831 & 1,300 & 6.8 & 18.0 & 15.0 & 322 \\
\hline 16 & 350721106222101 & $04-13-95$ & 2 & 3 & 43 & 432 & 730 & 9.3 & 18.5 & 14.0 & 312 \\
\hline 17 & 350949106184501 & $04-14-95$ & 1 & 330 & 0.6 & 424 & 713 & 7.3 & 20.5 & 13.5 & 286 \\
\hline 18 & 350655106185601 & $04-24-95$ & $<1$ & 710 & 1 & 976 & 1,750 & 6.7 & 12.0 & 13.0 & 274 \\
\hline 19 & 350522106222501 & $04-24-95$ & $<1$ & 620 & 1 & 948 & 1,530 & 7.1 & 10.0 & 12.0 & 288 \\
\hline 20 & 350525106151701 & $04-25-95$ & $<1$ & 820 & 0.9 & 1,120 & 2,090 & 7.0 & 12.5 & 8.0 & 198 \\
\hline 21 & 350620106192201 & $04-25-95$ & 4 & 220 & 2 & 466 & 800 & 7.3 & 13.0 & 15.0 & 286 \\
\hline 22 & 350739106195601 & $04-25-95$ & $<1$ & 670 & 1 & 935 & 1,440 & 6.9 & 15.0 & -- & 514 \\
\hline 23 & 350931106200901 & $04-26-95$ & $<1$ & 840 & 0.3 & 1,160 & 1,470 & 7.0 & 16.0 & 12.5 & 140 \\
\hline 24 & 350908106190601 & $04-26-95$ & 1 & 390 & 0.5 & 541 & 826 & 7.0 & 14.5 & 11.0 & 228 \\
\hline 25 & 350840106171601 & $04-26-95$ & $<1$ & 430 & 0.4 & 566 & 989 & 7.1 & 11.0 & 13.5 & 161 \\
\hline 26 & 350854106210001 & $04-28-95$ & $<1$ & 490 & 0.5 & 628 & 1,100 & 6.8 & 17.0 & 13.0 & 318 \\
\hline 27 & 350606106200401 & $05-30-95$ & $<1$ & 290 & 0.6 & 374 & 690 & 6.8 & 20.0 & 16.0 & 232 \\
\hline 28 & 350946106193501 & $05-31-95$ & 2 & 240 & 1 & 413 & 700 & 7.1 & 20.5 & 15.0 & 185 \\
\hline 29 & 350851106180301 & $05-31-95$ & $<1$ & 290 & 0.4 & 365 & 530 & 6.5 & 20.0 & 15.0 & 258 \\
\hline 30 & 350748106182601 & $05-31-95$ & $<1$ & 440 & 0.7 & 600 & 1,110 & 6.8 & 21.5 & 16.5 & 169 \\
\hline 31 & 350609106202201 & $06-01-95$ & 1 & 230 & 2 & 464 & 790 & 6.9 & 18.5 & 17.5 & 299 \\
\hline 32 & 350434106225701 & $06-01-95$ & $<1$ & 340 & 0.6 & 427 & 730 & 6.8 & 22.0 & 17.0 & 260 \\
\hline 33 & 345754106164601 & $06-02-95$ & $<1$ & 540 & 0.7 & 708 & 1,220 & 6.6 & 22.0 & 17.0 & 286 \\
\hline 34 & 345855106203601 & $06-02-95$ & $<1$ & 440 & 0.7 & 583 & 989 & 6.5 & 21.0 & 12.5 & 296 \\
\hline 35 & 350623106215301 & $06-02-95$ & $<1$ & 1,800 & 3 & 3,210 & 385 & 6.6 & 32.0 & 14.5 & 496 \\
\hline $\begin{array}{l}36 \\
37\end{array}$ & $\begin{array}{l}350735106223001 \\
350802106215401\end{array}$ & $\begin{array}{l}06-06-95 \\
06-06-95\end{array}$ & $\begin{array}{r}2 \\
<1\end{array}$ & $\begin{array}{l}270 \\
700\end{array}$ & $\begin{array}{l}1 \\
0.7\end{array}$ & $\begin{array}{l}433 \\
937\end{array}$ & $\begin{array}{r}760 \\
1,570\end{array}$ & $\begin{array}{l}6.7 \\
6.7\end{array}$ & $\begin{array}{l}21.5 \\
26.0\end{array}$ & $\begin{array}{l}14.0 \\
15.0\end{array}$ & $\begin{array}{l}287 \\
213\end{array}$ \\
\hline $1_{38}$ & 350610106223501 & 06-06-95 & $<1$ & 210 & 0.2 & 229 & 421 & 7.6 & 24.0 & 19.0 & 202 \\
\hline $\begin{array}{l}39 \\
40\end{array}$ & $\begin{array}{l}350557106182701 \\
350812106220001\end{array}$ & $\begin{array}{l}06-07-95 \\
06-07-95\end{array}$ & $\begin{array}{r}<1 \\
2\end{array}$ & $\begin{array}{l}660 \\
340\end{array}$ & $\begin{array}{l}0.7 \\
0.6\end{array}$ & $\begin{array}{l}894 \\
445\end{array}$ & $\begin{array}{r}1,510 \\
768\end{array}$ & $\begin{array}{l}6.8 \\
6.7\end{array}$ & $\begin{array}{l}21.5 \\
22.5\end{array}$ & $\begin{array}{l}18.5 \\
14.0\end{array}$ & 255 \\
\hline 41 & 350805106220801 & $06-07-95$ & 1 & 390 & 0.7 & 526 & 905 & 6.7 & 23.0 & 14.0 & 275 \\
\hline 42 & 345858106194601 & $06-08-95$ & $<1$ & 530 & 0.5 & 663 & 1,180 & 6.5 & 16.5 & 13.0 & 314 \\
\hline 43 & 350831106192301 & $06-08-95$ & $<1$ & 380 & 4 & 931 & 451 & 6.9 & 19.0 & 16.0 & 510 \\
\hline 244 & 350617106231101 & $06-08-95$ & $<1$ & 250 & 0.2 & 287 & 484 & 6.8 & 20.0 & 14.5 & 250 \\
\hline 245 & 350613106230601 & $06-08-95$ & $<1$ & 260 & 0.2 & 290 & 486 & 7.0 & 20.0 & 15.0 & 243 \\
\hline 46 & 350602106224701 & $06-09-95$ & $<1$ & 330 & 1 & 522 & 842 & 6.8 & 13.0 & 17.0 & 318 \\
\hline 47 & 350702106203101 & $06-12-95$ & $<1$ & 1,300 & 3 & 2,150 & 2,960 & 6.6 & 28.0 & 16.0 & 448 \\
\hline 48 & 345948106191701 & $06-12-95$ & $<1$ & 440 & 0.5 & 516 & 872 & 6.9 & 27.0 & 15.0 & 342 \\
\hline 49 & 350721106214101 & $06-14-95$ & $<1$ & 1,700 & 0.5 & 2,150 & 2,580 & 6.4 & 22.0 & 17.0 & 410 \\
\hline 50 & 350930106203001 & $06-14-95$ & 1 & 230 & 0.3 & 290 & 508 & 6.8 & 32.0 & 18.0 & 210 \\
\hline 51 & 350658106174401 & $06-14-95$ & $<1$ & 900 & 2 & 1,360 & 2,400 & 6.6 & 31.0 & 18.0 & 220 \\
\hline 52 & 350601106173101 & $07-25-95$ & $<1$ & 1,400 & 0.9 & 1,830 & 2,790 & 7.2 & 31.0 & 16.5 & 270 \\
\hline 53 & 350859106214301 & $07-26-95$ & $<1$ & 580 & 0.4 & 709 & 1,280 & 7.7 & 29.5 & 16.0 & 245 \\
\hline 54 & 350825106211101 & $07-27-95$ & $<1$ & 14 & 33 & 761 & 1,240 & 8.4 & 32.0 & 19.0 & 494 \\
\hline
\end{tabular}


Table 1.--Water-quality and ground-water-level data for wells, springs, and an acequia in the east mountain area, Bernalillo County, New Mexico, 1995--Concluded

\begin{tabular}{|c|c|c|c|c|c|}
\hline $\begin{array}{l}\text { site } \\
\text { number } \\
\text { (figure } \\
\text { 2) }\end{array}$ & $\begin{array}{c}\text { Station } \\
\text { 1dentification } \\
\text { number }\end{array}$ & $\begin{array}{c}\text { Date } \\
\text { sampled }\end{array}$ & $\begin{array}{l}\text { Land-surface } \\
\text { alt1tude } \\
\text { (feet above } \\
\text { sea level) }\end{array}$ & $\begin{array}{c}\text { Depth to } \\
\text { water } \\
\text { (feet below } \\
\text { land surface) }\end{array}$ & $\begin{array}{l}\text { Total depth } \\
\text { of well } \\
\text { (feet) }\end{array}$ \\
\hline $\begin{array}{l}1 \\
2\end{array}$ & $\begin{array}{l}350449106231901 \\
350423106263301\end{array}$ & $\begin{array}{l}03-14-95 \\
03-17-95\end{array}$ & $\begin{array}{l}6,355 \\
6,255\end{array}$ & $\begin{array}{l}26.17 \\
40.84\end{array}$ & $-\overline{146}$ \\
\hline 3 & 350604106205801 & $03-22-95$ & 6,520 & - & - \\
\hline 4 & 351014106202801 & $03-23-95$ & 6,798 & -- & - \\
\hline 5 & 350930106210701 & $03-23-95$ & 6,860 & - & - \\
\hline 6 & 351011106220401 & $03-24-95$ & 7,100 & 12.28 & -- \\
\hline 7 & 345918106202001 & $03-28-95$ & 7,660 & - & - \\
\hline 8 & 350410106262601 & $03-28-95$ & 6,030 & -- & -- \\
\hline 9 & 345833106185101 & $03-28-95$ & 7,420 & -- & - \\
\hline 10 & 350119106210901 & $03-29-95$ & 7,060 & -- & -- \\
\hline 11 & 345319106135101 & $03-31-95$ & 6,790 & -- & -- \\
\hline 12 & 350356106162901 & $03-31-95$ & 7,030 & - & -- \\
\hline 13 & 350615106223301 & $04-05-95$ & 6,580 & -- & -- \\
\hline 14 & 350949106211801 & $04-12-95$ & 6,940 & 188.61 & -- \\
\hline 15 & 350531106224301 & $04-12-95$ & 6,540 & 41.52 & 160 \\
\hline 16 & 350721106222101 & $04-13-95$ & 6,765 & 60.80 & 200 \\
\hline 17 & 350949106184501 & $04-14-95$ & 6,700 & - & $\ldots$ \\
\hline 18 & 350655106185601 & $04-24-95$ & 6,880 & - & -- \\
\hline 19 & 350522106222501 & $04-24-95$ & 6,400 & - & - \\
\hline 20 & 350525106151701 & $04-25-95$ & 6,775 & 141.42 & 275 \\
\hline 21 & 350620106192201 & $04-25-95$ & 6,880 & -- & -- \\
\hline 22 & 350739106195601 & $04-25-95$ & 6,800 & - & - \\
\hline 23 & 350931106200901 & $04-26-95$ & 6,740 & -- & -- \\
\hline 24 & 350908106190601 & $04-26-95$ & 6,800 & 155.07 & 178 \\
\hline 25 & 350840106171601 & $04-26-95$ & 6,780 & 109 & 20 \\
\hline 26 & 350854106210001 & $04-28-95$ & 6,960 & - & 450 \\
\hline 27 & 350606106200401 & $05-30-95$ & 6,730 & -- & - \\
\hline 28 & 350946106193501 & $05-31-95$ & 6,690 & 109.80 & -- \\
\hline 29 & 350851106180301 & $05-31-95$ & 6,720 & - & 185 \\
\hline 30 & 350748106182601 & $05-31-95$ & 6,880 & - & - \\
\hline 31 & 350609106202201 & $06-01-95$ & 6,580 & - & 137 \\
\hline 32 & 350434106225701 & $06-01-95$ & 6,350 & $-37.5 A$ & 253 \\
\hline 33 & 345754106164601 & $06-02-95$ & 7,220 & - & 125 \\
\hline 34 & 345855106203601 & $06-02-95$ & 7,570 & -- & -- \\
\hline 35 & 350623106215301 & $06-02-95$ & 6,540 & - & 225 \\
\hline 36 & 350735106223001 & $06-06-95$ & 6,840 & 51.70 & 245 \\
\hline 37 & 350802106215401 & $06-06-95$ & 6,855 & 37.90 & 200 \\
\hline $1_{38}$ & 350610106223501 & $06-06-95$ & 6,580 & NA & NA \\
\hline 39 & 350557106182701 & $06-07-95$ & 7,200 & - & - \\
\hline 40 & 350812106220001 & $06-07-95$ & 6,860 & 38.30 & 100 \\
\hline 41 & 350805106220801 & $06-07-95$ & 6,900 & 74.80 & -- \\
\hline 42 & 345858106194601 & $06-08-95$ & 7,490 & 32.40 & 100 \\
\hline 43 & 350831106192301 & $06-08-95$ & 6,886 & -- & -- \\
\hline 244 & 350617106231101 & $06-08-95$ & 6,800 & NA & NA \\
\hline 245 & 350613106230601 & $06-08-95$ & 6,760 & NA & NA \\
\hline 46 & 350602106224701 & $06-09-95$ & 6,600 & 41.74 & -- \\
\hline 47 & 350702106203101 & $06-12-95$ & 6,660 & 140 & -- \\
\hline 48 & 345948106191701 & $06-12-95$ & 7,680 & - & -- \\
\hline 49 & 350721106214101 & $06-14-95$ & 7,300 & - & -- \\
\hline 50 & 350930106203001 & $06-14-95$ & 6,810 & - & -- \\
\hline 51 & 350658106174401 & $06-14-95$ & 6,990 & 98.54 & 205 \\
\hline 52 & 350601106173101 & $07-25-95$ & 6,960 & - & $\ldots$ \\
\hline 53 & 350859106214301 & $07-26-95$ & 6,980 & - & -- \\
\hline 54 & 350825106211101 & $07-27-95$ & 6,920 & 125.00 & 150 \\
\hline
\end{tabular}

${ }^{1}$ Acequia
${ }^{2}$ Spring 


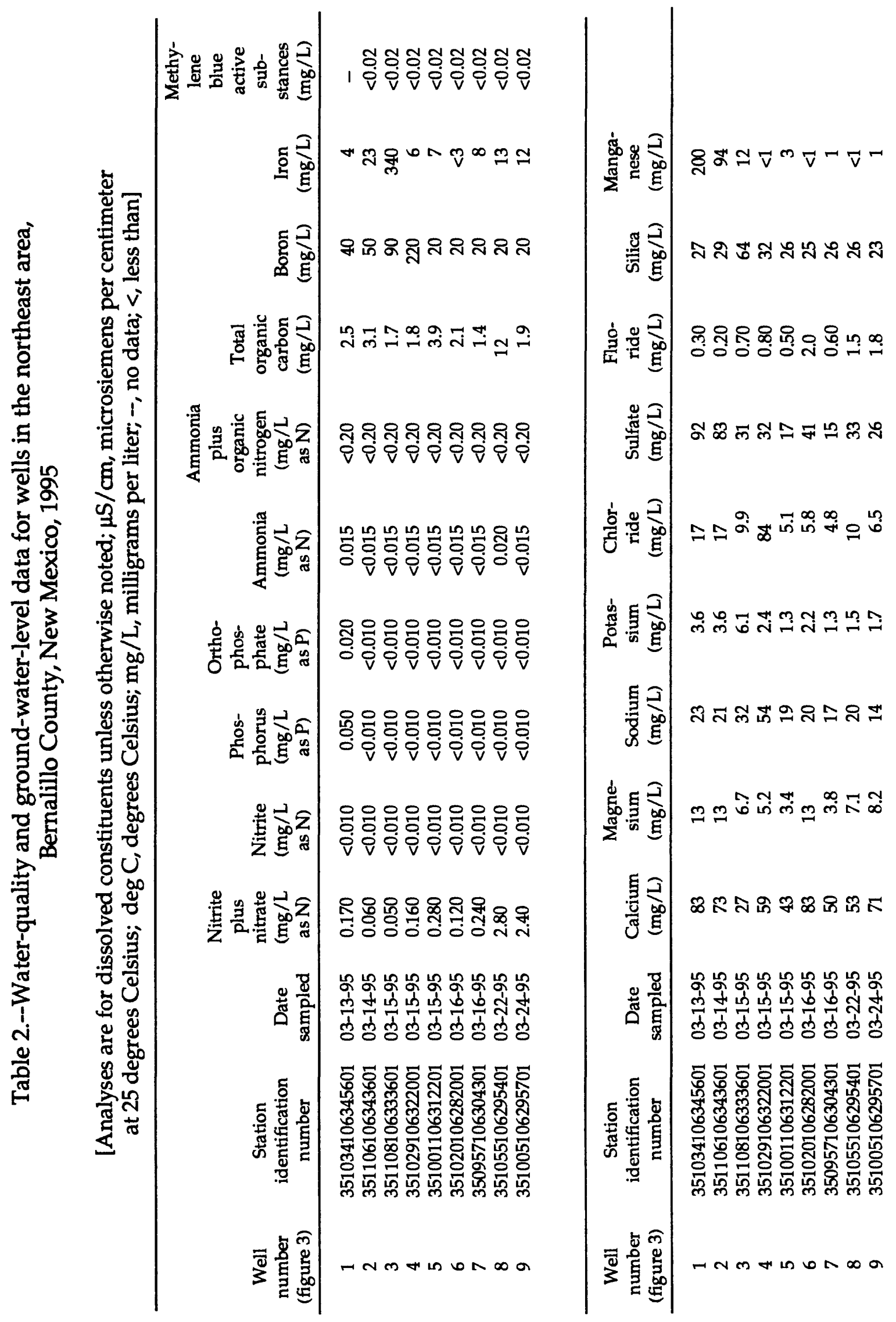




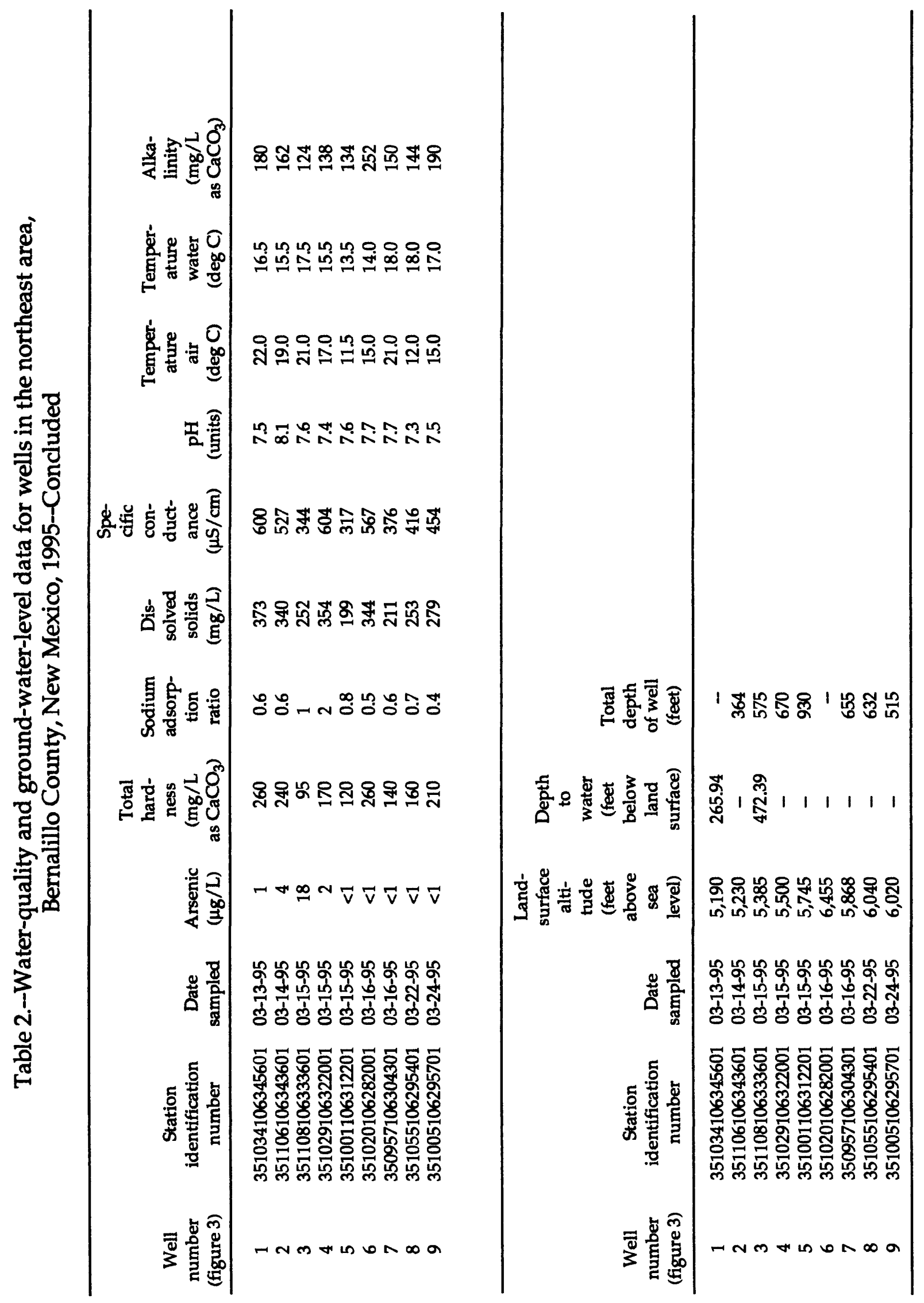

